\title{
Reactivity of various leishmanial antigens in a direct agglutination test and their value in differentiating post-kala azar dermal leishmaniasis from leprosy and other skin conditions
}

\author{
A. EL HARITH, S. CHOWDHURY* $\dagger$, A. AL-MASUM*, S. SEMIÃO-SANTOS, P. K. DAS $\ddagger, S$. AKHTER*, \\ J. C. M. VETTER and I. HAQ*
}

Department of Medical Microbiology, Academic Medical Centre, University of Amsterdam, the Netherlands, *Institute of Epidemiology Disease Control and Research, Mohakhali, Dhaka-1212, †National Institute of Preventive and Social Medicine, Mohakhali, Dhaka-1212, Bangladesh and $\ddagger$ Department of Pathology/ Dermatology, Academic Medical Centre, University of Amsterdam, the Netherlands

\begin{abstract}
A direct agglutination test (DAT) for the detection of post-kala azar dermal leishmaniasis (PKDL) was evaluated in conditions that simulate the disease clinically or immunologically. A reference strain of Leishmania donovani (LEM 1399), and antigen preparations from two Leishmania isolates from Bangladeshi patients with post-kala azar dermal leishmaniasis or visceral leismaniasis were used. A titre of at least 51200 was obtained in tests of patients with PKDL with all three antigens, whereas a maximum titre of 1600 was recorded in patients with cutaneous leishmaniasis, mucocutaneous leishmaniasis or leprosy. Antigens from dermal isolates of $L$. tropica (LV 140) and L. braziliensis (LV 65) yielded titres of 1600-6400 in patients with PKDL. The lowest titre recorded in $\mathbf{7 0}$ patients tested with the homologous PKDL antigen was 409600. In patients with leprosy, cutaneous leishmaniasis, syphilis, onchocerciasis, tuberculosis, blastomycosis or vitiligo, titres ranged from 100 to 1600 . The DAT is better than current parasitological and histopathological methods for the diagnosis of PKDL in areas in which leprosy is co-endemic.
\end{abstract}

\section{Introduction}

As well as being the main source of transmission of visceral leishmaniasis, post-kala azar dermal leishmaniasis (PKDL) is easily confused with leprosy in the Indian subcontinent and East Africa [1-3]. Absence of leishmania parasites in skin lesions of patients with PKDL may lead to a misdiagnosis of leprosy, especially as impaired organ sensation may occur in PKDL through involvement of dermal nerves [4]. The mononuclear cell infiltration of peripheral nerves characteristic of lepromatous leprosy may contribute to further confusion in the histopathological diagnosis of PKDL. Exclusion of other dermal abnormalities due to cutaneous and mucocutaneous leishmaniasis, syphilis, yaws, blastomycosis and other fungal infections is also imperative for the correct diagnosis [5]. Previous history of visceral leishmaniasis, although important, could be misleading since such infection may have been asymptomatic or subclinical $[3,5]$.

Demonstration of the causative micro-organisms in

Received 13 June 1995; revised version accepted 14 Aug. 1995.

Corresponding author: A. El Harith.
PKDL and leprosy is impeded by the insensitivity of present laboratory methods [2]. Despite recent improvements, differentiation between the two infections by antibody detection or cellular stimulation techniques is still lacking [5]. Use of a different panel of antigen preparations in the leishmanin skin test did not improve the specificity of the test. Serum IgG and IgM antibodies revert to normal after resolution of visceral leishmaniasis and may be hardly measurable in the immunofluorescence (IFAT) or aldehyde tests in patients with PKDL [6].

Previous results with an improved direct agglutination test (DAT) showed a satisfactory sensitivity and specificity for the routine diagnosis of visceral and canine leishmaniasis $[7,8]$. The sensitivity of DAT for the detection of anti-leishmania antibodies in parasitologically or clinically diagnosed cases of PKDL by use of antigens from a reference strain and two isolates from Bangladesh has now been evaluated. The specificity of the test in dermal conditions known to be clinically indistinguishable from PKDL was also assessed. Because of their immunological relationship, leishmanial antigens prepared from cases of visceral leishmaniasis and PKDL were compared for reactivity in DAT with sera from both patient groups. 


\section{Patients and methods}

\section{Antigens}

L. donovani MHOM/SD/OO/LEM 1399, L. tropica MHOM/IL/75/LV-140, L. braziliensis MHOM/BR/OO/ LV-65 and two uncharacterised Bangladeshi strains from the bone marrow of patients with kala azar (IEDCR-131) or nodular skin lesions of PKDL (IEDCR-3) were maintained in vitro in Novy, MacNeal and Nicolle's (NNN) or brain heart infusion culture media. For preparation of DAT antigen, promastigotes were mass cultured in RPMI-1640 supplemented with fetal calf serum (FCS) $15 \%$. After repeated washing in Locke's solution ( $\mathrm{pH} 7.6$ ), promastigote pellets were treated with trypsin as described previously [7]. The comparative effects of trypsin and 2-mercaptoethanol in the preparation of antigen were tested with the indigenous PKDL isolate (IEDCR-3). Logarithmic phase promastigotes were washed and suspended at a density of $10^{8} / \mathrm{ml}$ in Locke's solution ( $\mathrm{pH} \mathrm{7.6)} \mathrm{con-}$ taining 2-mercaptoethanol $1.2 \% \mathrm{v} / \mathrm{v}$ or trypsin. Promastigotes so treated were incubated at $37^{\circ} \mathrm{C}$ for $45 \mathrm{~min}$, washed and fixed for $18 \mathrm{~h}$ in Locke's solution supplemented with formaldehyde $2 \% \mathrm{w} / \mathrm{v}$. After fixation, parasites were stained with Coomassie Brilliant Blue and finally resuspended $\left(5 \times 10^{7} / \mathrm{ml}\right)$ in formaldehyde $0.4 \% \mathrm{w} / \mathrm{v}$ citrate-saline as described elsewhere [7].

\section{Patients}

Blood samples were collected by venepuncture for sera or by finger-prick on to filter-paper (Whatman no. 3) from individuals with the following diagnoses.

Post-kala azar dermal leishmaniasis (PKDL). A group of 15 cases confirmed by demonstration of leishmania parasites at the Institute of Epidemiology, Disease Control and Research (IEDCR)-Dhaka, Bangladesh; serum samples were collected before or during treatment.

A second group of 70 patients identified during a cross-sectional survey of visceral leishmaniasis in Bangladesh, covering an endemic population of 37954 inhabitants [9]. PKDL was diagnosed either by demonstration of parasites in skin slit smears (42 cases) or clinically (28 cases) on the basis of symptoms and normal organ sensation to exclude leprosy and visceral leishmaniasis. Most patients were from Mymensingh (32 patients) and Sirajganj (18 patients); 20 were from Pabna, Bogra, Comilla, Manikgong, Gazipur, Chaudpur, Patuakhali, Laximipur and Dhaka districts. Air-dried blood spots were packed in polythene bags and mailed to the collaborating laboratory at the University of Amsterdam.

Visceral leishmaniasis. Thirty-two cases from Mymensingh district diagnosed by bone marrow aspiration at IEDCR. Serum samples were collected either before or during administration of sodium antimony gluconate.
Cutaneous leishmaniasis. Parasitologically confirmed cases from Algeria (24 cases) and Brazil (24 cases) diagnosed at Institut Pasteur D'Algier (Algiers), Instituto Lauro de Souza Lima (São Paulo) and Instituto Alfredo da Matta (Manaus-Amazónia); serum samples were donated by R. Belazzoug, F. Morosa and D. Opramolla.

Mucocutaneous leishmaniasis. Parasitologically diagnosed cases from Bolivia (five cases) and Brazil (three); sera were provided by P. Desjeux (Institut Pasteur, Paris).

Leprosy. Patients were diagnosed clinically or histopathologically by demonstration of Mycobacterium leprae in skin, nasal mucous membrane or nerve biopsy specimens. Sera from 27 patients (20 from the Netherlands, seven from the Philippines) were donated by $\mathrm{P}$. Klatser (Royal Tropical Institute, Amsterdam). Thirty-two samples were obtained from patients diagnosed during collaborative studies in Spain (10), Surinam (six), Thailand (three), Morocco (three) or at the Academic Medical Centre in Amsterdam (10). Patients were classified according to the Ridley-Jopling scale [10]: 24 lepromatous, 19 border-line lepromatous, seven tuberculoid, six border-line tuberculoid and three border-line. All of these sera had high antibody levels to mycobacterial immuno-cross-reactive antigen(s) as demonstrated by both Western blotting and enzymelinked immunosorbent assay (ELISA) [11].

Syphilis. Dutch patients with active (24 patients) or treated (26 patients) Treponema pallidum infection diagnosed at the National Institute of Public Health and Environmental Protection, Bilthoven, the Netherlands (R. V. W. van Eijk).

Onchocerciasis. Confirmed cases from Sierra Leone with dermal presentation of nodular lesions (19 cases) or positive skin snip for microfilariae (16 cases). Diagnosis was established at Lunsar Eye Hospital by E. Rietveld and J. Stilma (Free University of Amsterdam). Venous blood for sera was collected before administration of chemotherapy.

Vitiligo. Five patients were diagnosed at the Department of Dermatology of the Academic Medical Centre, Amsterdam.

Other dermal conditions. Cutaneous tuberculosis (four cases) and psoriasis (three cases) diagnosed at the Academic Medical Centre or blastomycosis (two cases) at the Instituto Lauro de Souza Lima (São Paulo).

Tuberculosis. Sera from 33 culture- or X-rayconfirmed cases obtained from our serum bank. All samples showed high antibody levels to various $M$. leprae antigens $(80 \mathrm{kDa}, 66 \mathrm{kDa}, 58-60 \mathrm{kDa}, \quad 38$ $40 \mathrm{kDa}, \quad 45 \mathrm{kDa}, \quad 20-27 \mathrm{kDa}$ and $12-14 \mathrm{kDa})$ by Western blot technique and by ELISA with a crude 
water soluble fraction. The samples also contained high antibody levels to mycobacterial A60 antigens as demonstrated by ANDA Kit (Biologicals, StrassbourgFrance) [12].

Autoimmune disorders. Thirty-three Dutch nationals with positive rheumatoid factor (10), antimitochondrial (seven), anti-smooth muscle (seven) or antidouble stranded $\alpha$-DNA (nine) antibody tests. Sera were provided by $H$. G. M. Geertzen (Central Laboratory for Blood Transfusion), Amsterdam.

Controls. Eleven healthy individuals from Bangladesh.

\section{DAT procedures}

Sera were examined by the improved procedure described elsewhere [7]. Air-dried specimens collected by finger-prick on filter-paper were processed as follows: excised blood spots of $6 \mathrm{~mm}$ diameter, equivalent to $8.4 \mu \mathrm{l}$ serum content, were eluted overnight in $760 \mu \mathrm{l}$ of saline solution $(0.15 \mathrm{M} \mathrm{NaCl})$ at $4{ }^{\circ} \mathrm{C}$. According to a checkerboard titration procedure, the resultant eluate corresponded to a 1 in 100 serum dilution [13]. Heterologous samples showing agglutinating reactivity at 1 in 200 dilution or higher were retested with 2-mercaptoethanol $1.2 \% \mathrm{v} / \mathrm{v}$ and incubated for $1 \mathrm{~h}$ at $37^{\circ} \mathrm{C}$ [8]. After further incubation at room temperature $\left(21-22^{\circ} \mathrm{C}\right)$ for $18-20 \mathrm{~h}$, the DAT was read visually against a white background.

\section{Study design}

The value of DAT for detecting the antibody response in PKDL was assessed in 15 sera from confirmed cases. Specificity was determined by tests in dermal disorders with a clinical presentation resembling PKDL: 11 patients with cutaneous leishmaniasis, eight with mucocutaneous leishmaniasis and 10 with leprosy were included in this part of the study. Since PKDL can be associated with visceral or cutaneous leishmaniasis by virtue of the tropism of the parasite, reactivity of the indigenous PKDL antigen (IEDCR-3) was compared with that of the visceral (IEDCR-131 and LEM-1399) and cutaneous (LV-140 and LV-65) strains. Performance of the PKDL antigen was further evaluated in 70 whole blood samples from Bangladeshi patients with PKDL. Sera from 49 patients with different forms of leprosy, 37 with cutaneous leishmaniasis, 35 with onchocerciasis, 50 with syphilis, 33 with tuberculosis, three with psoriasis, two with blastomycosis, 33 with autoimmune disorders, five with vitiligo and 18 with kala azar were included to further confirm the specificity for the diagnosis of PKDL. A batch of antigen processed with 2mercaptoethanol was evaluated in those sera for which the DAT titres with the trypsinated antigen were $\geqslant 200$.

\section{Results}

High titres were obtained for all 15 PKDL sera in DAT assays with antigen from the reference strain (LEM 1399); similar results were obtained with the analogous visceral leishmaniasis isolate (IEDCR-131) (Fig. 1). In all five patient groups studied, titres obtained with the PKDL isolate, IEDCR-3, were more similar to those of the visceral (LEM-1399 and IEDCR-131) than the cutaneous (LV-140 and LV-65) strains of leishmania (Figs 1 and 2). Compared with visceral leishmaniasis and PKDL antigen preparations, those of cutaneous and mucocutaneous leishmaniasis yielded extremely low titres with serum samples from patients with kala azar or PKDL (Fig. 2). Even when applied to homologous sera from patients with cutaneous or mucocutaneous disease, titres obtained with the cutaneous antigens (LV-140 and LV-65) did not exceed 12800.

The indigenous PKDL (IEDCR-3) antigen was selected for comparison with antigen prepared from the reference strain LEM 1399 (Table 1). In none of the 70 PKDL patients examined was a titre below 409600 recorded with either of the two antigens. Titres obtained with the PKDL isolate were generally higher than those seen with the reference strain. No appreciable difference in titre level was observed between 42 confirmed and 28 clinically diagnosed cases.

Specificity of the PKDL antigen was further demonstrated by the remarkably low titres $(\leqslant 1600)$ seen in tests on patients with dermal conditions clinically simulating PKDL (Table 2).

Incorporation of 2-mercaptoethanol in antigen processing resulted in further improvement in the specificity of DAT for the detection of PKDL (Table 3). Titres ranging from 200 to 1600 obtained with the trypsinated antigen in patients suffering from leprosy or other dermal conditions were reduced to $\leqslant 200$.

\section{Discussion}

The potential of DAT in the diagnosis of PKDL is clearly demonstrated by the considerable titres obtained in confirmed and unconfirmed cases (Tables 1 and 2). The test was also specific in patients with dermal conditions difficult to separate from PKDL on clinical grounds. Many dermal features of PKDL such as hypopigmented macules, generalised nodules and papules, are also common in leprosy, psoriasis, syphilis, yaws and blastomycosis [2,5]. Furthermore, the depigmentation characterising some non-infectious dermatoses, as in vitiligo, may resemble early onset PKDL or leprosy. As a high level of clinical expertise is required to diagnose PKDL, a highly specific confirmatory test is valuable.

The DAT method described retained its specificity in 


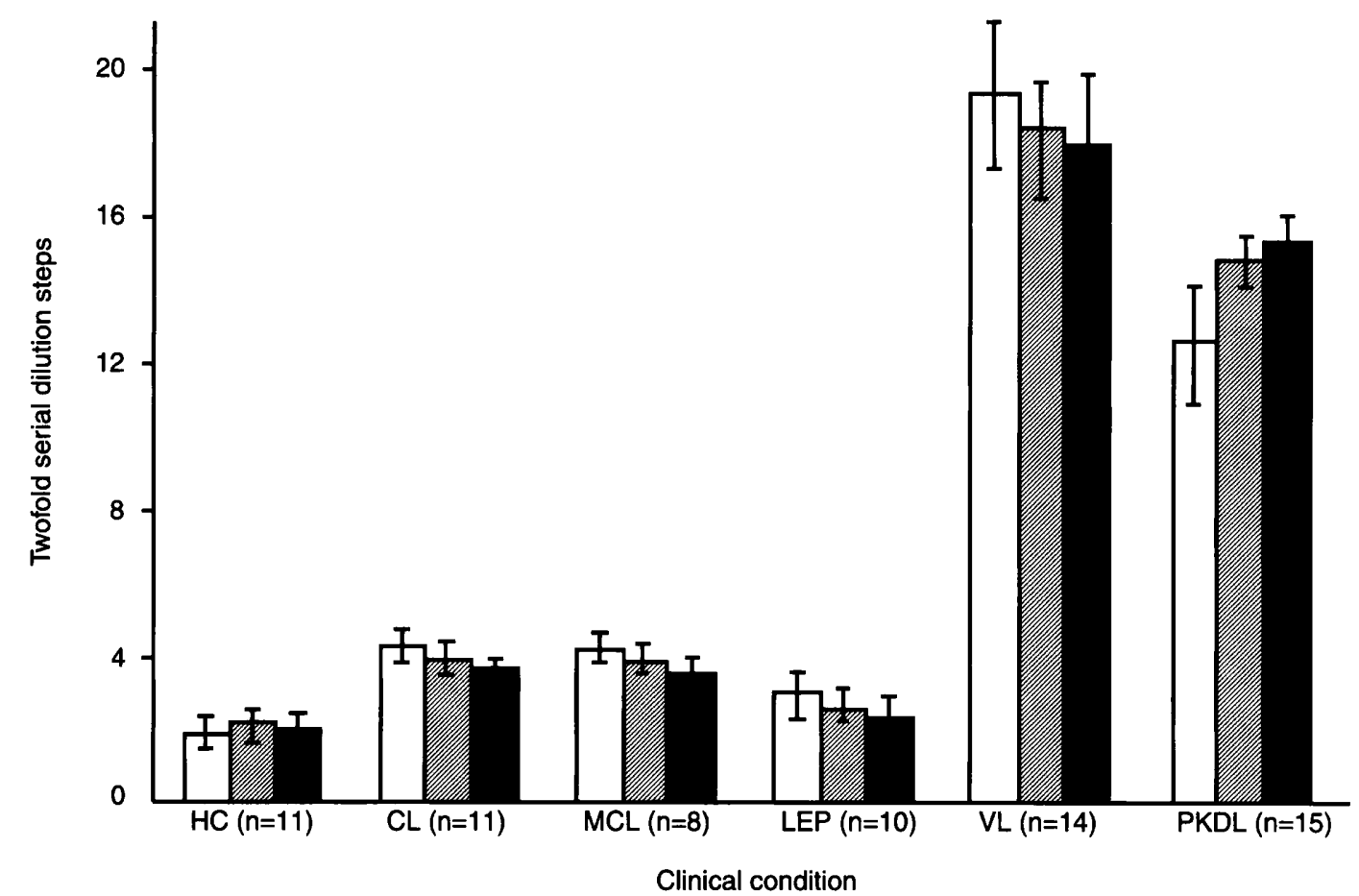

Fig. 1. Direct agglutination test (DAT) readings in patients with post-kala azar dermal leishmaniasis (PKDL), cutaneous (CL) and mucocutaneous (MCL) leishmaniasis, leprosy (LEP), visceral leishmaniasis (VL) or healthy controls (HC) tested against antigens from the reference strain (SD/LEM-1399; $\square$ ) and two indigenous isolates from VL (IEDCR131; $\triangle$ ) and PKDL (IEDCR-3; $\mathbf{0}$ ) Bengali cases. Readings are expressed as means and SD (bar) of two-fold serial dilutions from 1 in 100 to 1 in 26214400 (20 dilution steps).

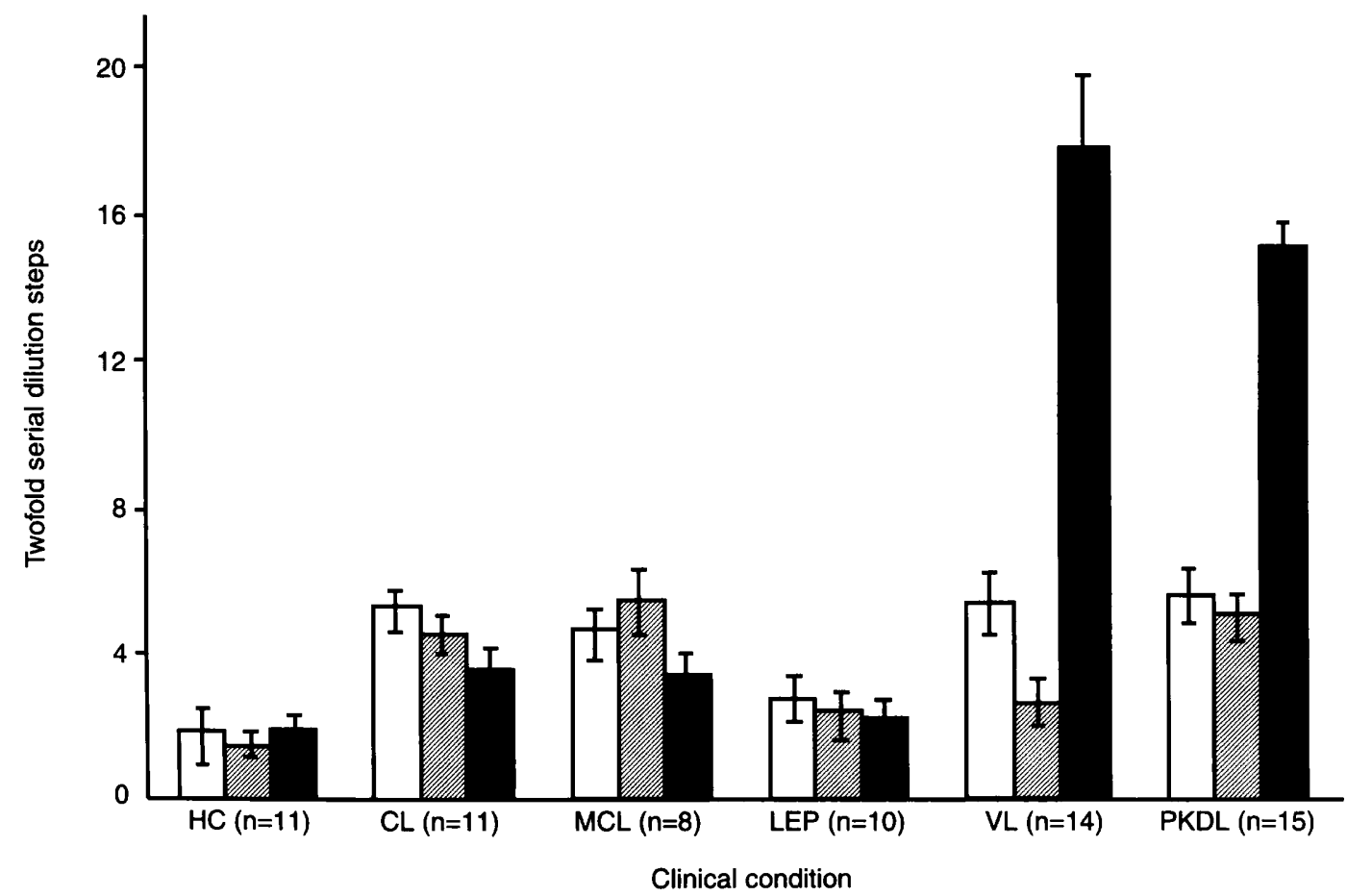

Fig. 2. Direct agglutination test (DAT) readings in patients with post-kala azar dermal leishmaniasis (PKDL), cutaneous (CL) and mucocutaneous (MCL) leishmaniasis, leprosy (LEP), visceral leishmaniasis (VL) or healthy controls (HC) tested against antigens from L. tropica (LV 140; $\square$ ), L. braziliensis (LV 65; $\square$ ) and an indigenous PKDL isolate from Bangladesh (IEDCR-3; ). Readings are expressed as means and SD (bar) of two-fold serial dilutions from 1 in 100 to 1 in 26214400 (20 dilution steps).

cutaneous and mucosal leishmaniasis caused by $L$. tropica and L. braziliensis. In these two forms of leishmaniasis, lesions are mostly confined to tegumental and mucosal tissues and an excessive cellular response eventually leads to development of allergic nodular lesions harbouring very few leishmania parasites, a condition mimicking PKDL and leprosy. Moreover, because of the appearance of metastatic lesions on the face, and massive ulceration of the nasal cartilage and nasopharynx, diffuse cutaneous 
Table 1. Reactivity of the indigenous (IEDCR-3) and reference (LEM 1399) Leishmania strain antigens against whole blood samples from patients with PKDL

\begin{tabular}{|c|c|c|c|c|c|c|c|c|c|}
\hline \multirow{2}{*}{$\begin{array}{l}\text { PKDL } \\
\text { diagnosis }\end{array}$} & \multirow{2}{*}{$\begin{array}{c}\text { Number of } \\
\text { patients }\end{array}$} & \multirow{2}{*}{$\begin{array}{l}\text { Antigen } \\
\text { preparation }\end{array}$} & \multicolumn{7}{|c|}{ DAT readings* } \\
\hline & & & 13 & 14 & 15 & 16 & 17 & 18 & $19-20$ \\
\hline \multirow[t]{2}{*}{ Parasitological } & 42 & IEDCR-3 & 1 & 2 & 3 & 19 & 10 & 4 & 3 \\
\hline & & LEM-1399 & 3 & 14 & 5 & 17 & 1 & 1 & 1 \\
\hline \multirow[t]{2}{*}{ Clinical } & 28 & IEDCR-3 & 0 & 1 & 2 & 15 & 5 & 2 & 3 \\
\hline & & LEM-1399 & 2 & 13 & 4 & 8 & 0 & 1 & 0 \\
\hline
\end{tabular}

${ }^{*}$ Expressed as the number of two-fold serial dilutions performed, representing titres from 409600 to 26214400 (13-20 dilutions).

Table 2. Titres obtained with the antigen preparation from the indigeneous Leishmania isolate, IEDCR-3, in sera of patients with PKDL or other conditions

\begin{tabular}{|c|c|c|c|c|c|c|}
\hline \multirow[b]{2}{*}{ Clinical condition (n) } & \multicolumn{6}{|c|}{ Number of patients with DAT titre of } \\
\hline & 100 & 200 & 400 & 800 & 1600 & $\geqslant 25600$ \\
\hline$\overline{\mathrm{CL} \text { (Algeria) (13) }}$ & 10 & 1 & 2 & 0 & 0 & 0 \\
\hline CL (Brazil) (24) & 18 & 2 & 3 & 1 & 0 & 0 \\
\hline Leprosy LL (17) & 10 & 2 & 2 & 3 & 0 & $\mathbf{0}$ \\
\hline BL (16) & 6 & 1 & 4 & 4 & 1 & 0 \\
\hline TT (7) & 7 & 0 & 0 & 0 & 0 & 0 \\
\hline BT (6) & 6 & 0 & 0 & 0 & 0 & 0 \\
\hline BB (3) & 3 & 0 & 0 & 0 & 0 & 0 \\
\hline Onchocerciasis (35) & 35 & 0 & 0 & 0 & 0 & 0 \\
\hline Syphilis $(50)$ & 43 & 2 & 2 & 3 & 0 & 0 \\
\hline Vitiligo (5) & 5 & 0 & 0 & 0 & 0 & 0 \\
\hline Psoriasis (3) & 3 & 0 & 0 & 0 & 0 & 0 \\
\hline Blastomycosis (2) & 2 & 0 & 0 & 0 & 0 & 0 \\
\hline Cutaneous tuberculosis (4) & 4 & 0 & 0 & 0 & 0 & 0 \\
\hline Pulmonary tuberculosis (33) & 31 & 2 & 0 & 0 & 0 & 0 \\
\hline Autoimmune disorders (33) & 26 & 5 & 2 & 0 & 0 & 0 \\
\hline VL (18) & 0 & 0 & 0 & 0 & 0 & 18 \\
\hline PKDL (70) & 0 & 0 & 0 & 0 & 0 & 70 \\
\hline
\end{tabular}

CL, cutaneous leishmaniasis; VL, visceral leishmaniasis; PKDL, post-kala azar dermal leishmaniasis; LL, lepromatous; BL, borderline lepromatous; TT, tuberculoid; BT, borderline tuberculoid; BB, borderline leprosy.

Table 3 Reactivity of trypsin- and 2-mercaptoethanol-processed antigen preparations of the PKDL strain (IEDCR-3) in tests of sera for which the initial DAT titre was $\geqslant 200$

\begin{tabular}{|c|c|c|c|}
\hline \multirow[b]{2}{*}{ Clinical condition } & \multirow[b]{2}{*}{ Number of sera } & \multicolumn{2}{|c|}{ Highest titre obtained with } \\
\hline & & Trypsin & 2-mercaptoethanol \\
\hline Healthy controls & $6(200-400)$ & 200 & $\leqslant 100$ \\
\hline CL (Algeria) & $3(200-400)$ & 100 & $\leqslant 100$ \\
\hline CL (Brazil) & $6(200-800)$ & 400 & $\leqslant 100$ \\
\hline Leprosy (LL) & $28(200-800)$ & 800 & $\leqslant 200$ \\
\hline Leprosy (BL) & $24(200-1600)$ & 800 & $\leqslant 200$ \\
\hline Syphilis & $7(200-800)$ & 800 & $\leqslant 100$ \\
\hline Pulmonary & $2(200)$ & 100 & $\leqslant 100$ \\
\hline Autoimmune & $7(200-400)$ & 400 & $\leqslant 100$ \\
\hline VL & $18(>51200)$ & $>51200$ & $>51200$ \\
\hline PKDL (controls) & $8(>51200)$ & $>51200$ & $>51200$ \\
\hline
\end{tabular}

See Table 2 for abbreviations.

leishmaniasis and mucocutaneous leishmaniasis may be confused with leprosy in South America and PKDL in Eastern Africa $[14,15]$. The DAT permitted PKDL to be clearly differentiated from these conditions especially when antigen from an indigenous isolate was used (Table 2).

The extremely low titres obtained in patients with cutaneous or mucocutaneous leishmaniasis, despite the use of homologous antigens, was unexpected. Previous results had indicated that the test was potentially useful in infections with L. aethiopica [15]. The present results are, nonetheless, in agreement with those reported for Sudanese patients infected with $L$. major [16]. Humoral immunity seems to be of less importance than cell-mediated immunity in the diagnosis of cutaneous and mucocutaneous leishmaniasis, as shown by the leishmanin skin test in South America [17]. However, use of the skin test did not improve the efficiency of detection of PKDL in the Sudan [3].

The DAT offers a significant improvement in sensitivity over the immunofluorescence and aldehyde tests [18] in the diagnosis of PKDL. Whether the humoral response measured is exclusively due to PKDL or is a 
sequel to primary visceral disease is difficult to verify. Studies in Kenya have shown that high DAT titres sometimes persisted for as long as 36 months after completion of Pentostam therapy in patients with kala azar [7]. As assessment of cure in those patients was mainly clinical, it is uncertain whether complete clearance of the parasites had taken place. Unsuccessful therapy or under-treatment have been claimed to initiate PKDL [3]. Persistent DAT positivity was also observed in dogs with long-standing $L$. infantum infection that did not respond to repeated treatment [19].

Although PKDL and visceral leishmaniasis are clinically separate entities the present results indicate a considerable serological relationship. The antibody response in both patient groups and the crossreactivity of the two corresponding antigens were very similar (Figs 1 and 2). Likewise, antigens of L. tropica and $L$. braziliensis gave low titres in tests of sera from patients with visceral leishmaniasis or PKDL. Iso-enzyme electrophoresis has revealed a similar zymodeme pattern in isolates from patients with these diseases [5], although cloned kinetoplast mini-circle DNA has been found to differ in hybridisation tests [20].

In India and Bangladesh, the prevalences of visceral leishmaniasis and PKDL are closely associated [21]. Recognition and treatment of PKDL would contribute significantly to the control of kala azar. The DAT method described here is simple and requires no special equipment [7]. Finger-prick samples were as reliable as serum, thus facilitating large scale application. Although the laboratory-adapted $L$. donovani strain LEM 1399 was previously employed for antigen preparation, the results indicate that indigenous Leishmania isolates can also be used.

Assuming that PKDL is a sequel to unresolved visceral disease, follow-up procedures are indicated in patients in whom DAT titres remain high after treatment. Insensitive and invasive parasitological procedures are unsuitable for conducting frequent follow-up, but the DAT provides a practical means for assessing recovery. The high sensitivity of DAT should facilitate the detection of early PKDL, prevent unnecessary tissue damage and possible organ deformation, and allow earlier, simpler treatment.

We thank J. Dankert and J. van der Noorda, Department of Medical Microbiology, University of Amsterdam; A. H. J. Kolk, P. Klatser and G. Jaarings, Laboratory of Tropical Hygiene, Royal Tropical Institute, Amsterdam; H. Groenendijk, I. C. Huig and N. Naafs, Faculty of Medicine, Erasmus University, Rotterdam; E. Rietveld and J. Stilma Free University of Amsterdam, the Netherlands; Sadeqa Tahera, NIPSOM and Enamul Karim, IEDCR, Dhaka, Bangladesh; D. Evans, London School of Hygiene and Tropical Medicine, London F. Morosa and D. V. A. Opramolla, Instituto Lauro de Souza Lima, São Paulo and Instituto Alfredo da Matta, Manaus-Amazónia, Brazil; R. V. W. van Eijk, National Insitute of Public Health and Environment Protection, Bilthoven; H. G. M. Geerzen, Central Laboratory for Blood Transfusion, Amsterdam, the Netherlands and
R. Belazzoug, Institut Pasteur D'Algier, Algeria for their kind cooperation. These investigations were financed by the Scientific and Technological Co-operation (ISC) of the European Communities under grant C11-CT90-0788.

\section{References}

1. Ramesh V, Saxena U, Misra RS, Mukherjee A. Post-kala-azar dermal leishmaniasis. A case report strikingly resembling lepromatous leprosy. Lepr $\operatorname{Rev} 1991$; 62: 217-221.

2. Ramesh V. On the differences between post-kala-azar dermal leishmaniasis and leprosy. Trop Doct 1994; 24: 120-121.

3. Zijlstra EE. Kala-azar in the Sudan: epidemiological and clinical studies. PhD Thesis, University of Amsterdam, the Netherlands, 1995.

4. Elhassan AM, Ali MS, Zijlstra EE, Eltoum IA, Ghalib HW, Ahmed HMA. Post-kala-azar dermal leishmaniasis in the Sudan: peripheral neural involvement. Int $J$ Dermatol 1992; 31: $400-403$.

5. Manson-Bahr PEC, Bell DR. Visceral leishmaniasis. In: Manson's tropical diseases, 19th edn. London, Baillière Tindall. 1987: 87-113.

6. Addy M, Nandy A. Ten years of kala-azar in west Bengal, part 1. Did post-kala-azar dermal leishmaniasis initiate the outbreak in 24-Parganas? Bull WHO 1992; 70: 341-346.

7. Harith AE, Kolk AHJ, Leeuwenburg $\mathrm{J}$ et al. Improvement of a direct agglutination test for field studies of visceral leishmaniasis. J Clin Microbiol 1988; 26: 1321-1325.

8. Harith AE, Slappendel RJ, Reiter I et al. Application of a direct agglutination test for detection of specific antileishmania antibodies in the canine reservoir. $J$ Clin Microbiol 1989; 27: 2252-2257.

9. Chowdhury MS, Harith AE, Al Masum A, Karim EA, Al Rahman A. Prevalence of agglutinating anti-leishmania antibodies in two multi-thousand Bengali communities. Zeitschr Parasitenkunde 1993; 79: 444-450.

10. Ridley DS, Jopling WH. Classification of leprosy according to immunity. A five-group system. Int $J$ Lepr Other Mycobact Dis 1966; 34: 255-273.

11. Das PK, Rambukkana A, Baas JG, Groothuis DG, Halperin M Enzyme-linked immunosorbent assay for distinguishing serological responses of lepromatous and tuberculoid leprosies to the 29/33-Kilodalton Doublet and 64-Kilodalton antigens of Mycobacterium tuberculosis. J Clin Microbiol 1990; 28: 379-382.

12. Van der Werf TS, Das PK, van Soolingen D, Young S, van der Mark TW, Van den Akker R. Sero-diagnosis of tuberculosis with $\mathrm{A} 60$ antigen enzyme-linked immunosorbent assay: failure in HIV-infected individuals in Ghana. Med Microbiol Immunol 1992; 181: 71-76.

13. Semião-Santos SJ, Harith AE, Ferreira E, Pires CA, Sousa C, Gusmão A. Évora district as a new focus for canine leishmaniasis in Portugal. Parasitol Res 1995; 81: 235-239.

14. Marsden PD. Mucosal leishmaniasis ("espundia" Escomel, 1911). Trans $R$ Soc Trop Med Hyg 1986; 80: 859-876.

15. Mengistu $G$, Kieseling $R$, Akuffo $H$. The value of a direct agglutination test in the diagnosis of cutaneous and visceral leishmaniasis in Ethiopia. Trans $R$ Soc Trop Med Hyg 1990; 84: 359-362.

16. El Safi SH, Evans DA. A comparison of the direct agglutination test and enzyme-linked immunosorbent assay in the sero-diagnosis of leishmaniasis in the Sudan. Trans $R$ Soc Trop Med Hyg 1989; 83: 334-337.

17. Nascimento MD, Alcântara-Neves NM, Muniz MEB, Nunes SF, Paranhos M, Pontes de Carvalho LC. Induction and modulation of the immune response to Leishmania by Montenegro's skin test. Trans $R$ Soc Trop Med Hyg 1993; 87: 91-93.

18. Nandy A, Addy M, Chowdhury AB. Leishmanial blepharoconjunctivitis. Trop Geogr Med 1991; 43: 303-306.

19. Slappendel RJ. Canine leishmaniasis. A review based on 95 cases in The Netherlands. Vet $Q$ 1988; 10: 11-16.

20. Das Gupta S, Ghosh DK, Majumder HK. A cloned kinetoplast DNA mini-circle fragment from a Leishmania spp. specific for post-kala-azar dermal leishmaniasis strains. Parasitol 1991; 102: $187-191$.

21. Thakur CP, Kumar K. Post kala-azar dermal leishmaniasis: a neglected aspect of kala-azar control programmes. Ann Trop Med Parasitol 1992; 86: 355-359. 